\title{
Disorder and interactions in quantum Hall ferromagnets: effects of disorder in Skyrmion physics
}

\author{
Jairo Sinova $^{a}$, A.H. MacDonald ${ }^{a}$ S. M. Girvin ${ }^{c}$ \\ ${ }^{a}$ Department of Physics, University of Texas, Austin, TX 78712, USA \\ ${ }^{b}$ Department of Physics, Yale University, New Haven, CT 06520 USA
}

\begin{abstract}
We present a Hartree-Fock study of the competition between disorder and interactions in quantum Hall ferromagnets near $\nu=1$. We find that the ground state at $\nu=1$ evolves with increasing interaction strength from a quasi-metallic paramagnet, to a partially spin-polarized ferromagnetic Anderson insulator, and to a fully spin-polarized ferromagnet with a charge gap. Away from $\nu=1$, the ground state evolves from a conventional Anderson insulator, to a conventional quasiparticle glass, and finally to a ferromagnetic Skyrmion quasiparticle glass. These different regimes can be measured in low-temperature transport and NMR experiments. We present calculations for the NMR spectra in different disorder regimes.
\end{abstract}

\section{INTRODUCTION}

At Landau level filling factor $\nu=1$ in the clean limit the ground state of a two-dimensional electron system (2DES) is a ferromagnet with a charge gap [1]. Away from $\nu=1$ the ground state is populated by the elementary charge excitations which are topologically charged spin textures (Skyrmions) containing more than one single spin flip. The existence and stability of such excitations has been verified experimentally through nuclear magnetic resonance (NMR) experiments [2]. Recent experiments have indicated that disorder (even in the weak limit) also plays a role in the observed behavior in a more detailed study of the NMR spectra at low temperatures [3,4]. The competition between interactions and disorder in the study of quantum Hall ferromagnets has often, but not always [5.6], been neglected partially because of the lack of easily manageable techniques which can deal with both at the same level.

Such competition can be studied using the HartreeFock (HF) approximation which has the advantage of being exact in both the non-interacting and the nondisordered limits [1,7]. We can summarize the results of our model calculations as follows (shown in Fig. 11). As the interaction strength is increased relative to disorder at $\nu=1$, the 2DES ground state suffers a phase transition from a paramagnetic to a ferromagnetic state. For the disorder models we use, the fully polarized state is reached when the Coulomb energy scale is approximately twice the Landau-level-broadening disorder energy scale. Away from $\nu=1$, screening by mobile charges reduces the importance of disorder and the system reaches maximal spin-polarization at smaller interaction strengths. The maximally polarized ground state at moderate interaction strengths is best described as a glass of localized conventional (Laughlin) quasiparticles formed in the $\nu=1$ fully polarized vacuum. Only for stronger interactions do we find a phase transition to a state with noncollinear magnetization in which the localized particles have Skyrmionic character. We also present NMR spec- tra calculations at $\nu=1$ in the different disorder regimes observed.

\section{FORMALISM}

The HF approximation allows the interplay between disorder and interactions to be addressed in an equal footing while retaining a simple independent-particle picture of the many-body ground state. In this section, we outline the basic formalism of HF approximation calculations in the LLL limit. Further details on this formalism can be found elsewhere [7,8].

Here we only consider lowest Landau level (LLL) states in expanding the HF Hamiltonian. Neglecting the frozen kinetic-energy degree of freedom, the Hamiltonian in second quantization is written as

$$
H=H_{I}+H_{d i s}+H_{Z},
$$

where $H_{I}$ is the normal Coulomb part of the Hamiltonian, $H_{d i s}$ is the external disorder part of the Hamiltonian

$$
H_{d i s}=\int d \mathbf{r} \sum_{\sigma} v_{E}(\mathbf{r}) \hat{\psi}_{\sigma}^{\dagger}(\mathbf{r}) \hat{\psi}_{\sigma}(\mathbf{r})
$$

and $H_{Z}$ is the Zeeman term

$$
H_{Z}=-\frac{1}{2} g \mu_{B} \int d \mathbf{r} \sum_{\sigma \sigma^{\prime}} \hat{\psi}_{\sigma^{\prime}}^{\dagger}\left(\mathbf{r}^{\prime}\right) \hat{\psi}_{\sigma}\left(\mathbf{r}^{\prime}\right) \vec{\tau}_{\sigma^{\prime} \sigma} \cdot \vec{B}(\vec{r}),
$$

with $\sigma=\uparrow, \downarrow, v_{E}$ being disorder potential, and $\tau_{i}$ being the Pauli matrices. The Zeeman coupling strength is given by $\tilde{g}=g \mu_{B} B /\left(e^{2} / \epsilon l\right)$. For $v_{E}(\mathbf{r})$ we use a white noise distribution so $\left\langle\left\langle v_{E}(\mathbf{r}) v_{E}\left(\mathbf{r}^{\prime}\right)\right\rangle\right\rangle=\sigma^{2} \delta\left(\mathbf{r}-\mathbf{r}^{\prime}\right)$. We define the parameter $\gamma \equiv e^{2} /(\in \sigma)$ as the ratio of the relative strength of interactions and disorder broadening. We chose the commonly used Landau gauge elliptic theta functions as our LLL basis to perform Hartree-Fock calculations in this system. 


\section{RESULTS}

At $\nu=1$ the ground state, in the disorder free limit, becomes a strong ferromagnet. Such collective behavior has been observed [2] in NMR Knight shift experiments in high quality samples. In figure 2 we present the $\mathrm{HF}$ theory results obtained for the dependence of the average magnitude of the spin polarization (insert) as a function of interaction strength and its corresponding NMR spectra at the lowest temperatures where there is no motional narrowing of the spectral line shape [4,9]. The Zeeman coupling strength $\tilde{g}=0.015$ is chosen to match the experimental values. Such NMR intensity spectrum is given by

$$
I(f, \gamma) \propto \int d \mathbf{r} \rho_{N}(z) e^{-\frac{1}{2 \sigma^{2}}\left(2 \pi f-2 \pi K_{s} \rho_{e}(z)\langle\vec{S}(\mathbf{r} ; \gamma)\rangle\right)},
$$

with $\sigma=9.34 \mathrm{~ms}^{-1}$ and $K_{s} \sim 25 \mathrm{KHz}$. Here $\rho_{N}(z)$ is the nuclear polarization density and $\rho_{e}(z)$ is the electron density envelope function in the quantum well. The evaluation of such spectra has been outlined elsewhere [4,9]. The partially polarized region shows a very distinct spectra which may explain the existence of anomalous spectra line shapes in certain samples where estimates of the disorder broadening through mobility measurements 10 indicate the possibility of being in such regime.

The partially polarized regime can also be studied experimentally by measuring the transport activation gap. Provided that weak Zeeman coupling can be ignored the Hall conductivity should jump from 0 to $2 e^{2} / h$ at $\nu=1$. In the ferromagnetic state, the majority-spin extended quasiparticle state will be below the Fermi level and the Hall conductivity at $\nu=1$ should be quantized at $\sigma_{x y}=e^{2} / h$. This spontaneous splitting is experimentally accessible and should exhibit interesting non-trivial power law critical behavior as the ferromagnetic state is entered. One important feature that our calculations demonstrate is that charge-density fluctuations at $\nu=1$ do not necessarily require the presence of well-defined Skyrmion quasiparticles.

In the strong disorder limit spontaneous spin polarization does not occur at any filling factor near $\nu=1$. However, in the large $\gamma$ (clean) limit, where full polarization is observed at $\nu=1$, the global polarization decays rapidly with $|1-\nu|$ as observed experimentally [2]. The global polarization results for $\nu \neq 1$ in Fig. 3, illustrate how the system interpolates between these two extrema. As the interaction strength $\gamma$ is increased from 0 to 2, the behavior mirrors the $\nu=1$ case. For strong disorder charge fluctuations dominate, and small spin polarizations occur primarily because many single particle orbitals are simultaneously occupied by both up and down spin electrons. In this regime charge variation is the main response to disorder and continues to play an important role at all interaction strengths. At sufficiently large $\gamma$, our finite size systems reach a state with the maximum spin polarization allowed by the Pauli exclusion principle. This maximally polarized state is reached earlier than in the case at $\nu=1$ ( $\gamma \sim 1.4-1.6)$ because, we believe, a larger number of charged quasiparticles are available to screen the random potential. At this point the system forms what we refer to as a conventional quasiparticle glass (CQG). The conventional Laughlin quasiparticles are initially localized in the deepest minima (or maxima for $\nu<1$ ) of the effective disorder potential and as the interaction strength increases, the charged quasiparticles rearrange themselves locally into a quasi-triangular Wigner crystal pinned by the strongest of the disorder potential extrema. At larger $\gamma$ there is a marked reduction of the global polarization from its maximally polarized value. This indicates a transition from a CQG to a Skyrmion glass (here glass is meant as a non-Bravis lattice arrangement of the Skyrminos). The point of cross over from the CQG to the Skyrmion glass, as illustrated in Fig. 3, depends on filling factor and $\tilde{g}$. We approximate the dependence of the transition point on $\tilde{g}$ in this regime by considering a simple model for a single Skyrmion trapped at a disorder potential extrema. We approximate its energy by

$$
E(K)=U\left(K-K_{0}\right)^{2}+g^{*} \mu_{B} B K+\sigma A K,
$$

where $K$ is the number of spin flips per Skyrmion, and $A$ is a phenomenological parameter. The first two terms pin the optimal Skyrmion size in the clean limit [11]. The third term favors the stronger localization of reduced (small $K$ ) Skyrminos close to the potential extrema. This simple model gives an estimate of the interaction strength at which $K>0$ Skyrmions first become stable

$$
\gamma^{*}=\frac{A}{2 K_{0} U /\left(e^{2} / \epsilon \ell\right)-\tilde{g}} .
$$

At factor $\nu=1.25, U /\left(e^{2} / \epsilon \ell\right) \sim 0.014$ and $K_{0} \sim 1$, $A \sim 0.1$ [7, 11. From this, one obtains an estimate of $\gamma^{*} \sim 7$ for the cross over point from conventional quasiparticles to Skyrmions at $\tilde{g}=0.015$. This is in reasonable agreement with the actual cross over point $\gamma^{*} \sim 10$ (see Fig. 3. In the clean limit, the Skyrmion system crystallizes in a square lattice for the filling factors considered here. (The Skyrmion crystal is triangular [11] for $\nu$ very close to 1.) These estimates of the maximum disorder strength at which Skyrmion physics is realized could be checked by performing NMR experiments in samples where electron density can be adjusted through gate voltages.

\section{ACKNOWLEDGEMENTS}

This work was supported by the Welch Foundation, and NSF grants DMR-9714055 and DMR-9820816. 
[1] S.M. Girvin, The Quantum Hall Effect: Novel Excitations and Broken Symmetries in Les Houches Summer School 1998 (to be published by Springer Verleg and Les Editions de Physique, 1999), and references therein.

[2] R. Tycko, S. E. Barrett, G. Dabbagh, L. N. Pfeiffer,and K.W. West, Science 268, 1460 (1995); S. E. Barrett, G. Dabbagh, L. N. Pfeiffer, K.W. West, and R. Tycko, Phys. Rev. Lett. 74, 5112 (1995).

[3] P. Khandelwal, A.E. Dementyev, N.N. Kuzma, S.E. Barrett, L.N. Pfeiffer, and K.W. West, e-print:condmat/0009134.

[4] J. Sinova, S. M. Girvin, T. Jungwirth, and K. Moon, Phys. Rev. B 61, 2749 (2000).

[5] A. G. Green, Phys. Rev. B 57, R9373 (1998).

[6] A. J. Nederveen and Yuli V. Nazarov, Phys. Rev. Lett. 82, 406 (1999).

[7] J. Sinova, S. M. Girvin, T. Jungwirth, and K. Moon, Phys. Rev. B 62, 13579 (2001).

[8] S.-R. Eric Yang and A.H. MacDonald, Phys. Rev. Lett. 70, 4110 (1993); S.-R. Eric Yang, A.H. MacDonald, and Bodo Huckestein, Phys. Rev. Lett. 74, 3229 (1995).

[9] N.N. Kuzma, P. Khandelwal, S.E. Barrett, L.N. Pfeiffer, and K.W. West, Science 281, 686 (1998); P. Khandelwal, N.N. Kuzma, S.E. Barrett, L.N. Pfeiffer, and K.W. West, Phys. Rev. Lett. 81, 673 (1998)

[10] T. Ando and Y. Uemura, J. Phys. Soc. Japan 36, 959 (1974).

[11] R. Côté, A.H. MacDonald, L. Brey, H.A. Fertig, S.M. Girvin, and H.T.C. Stoof, Phys. Rev. Lett. 78, 4825 (1997).

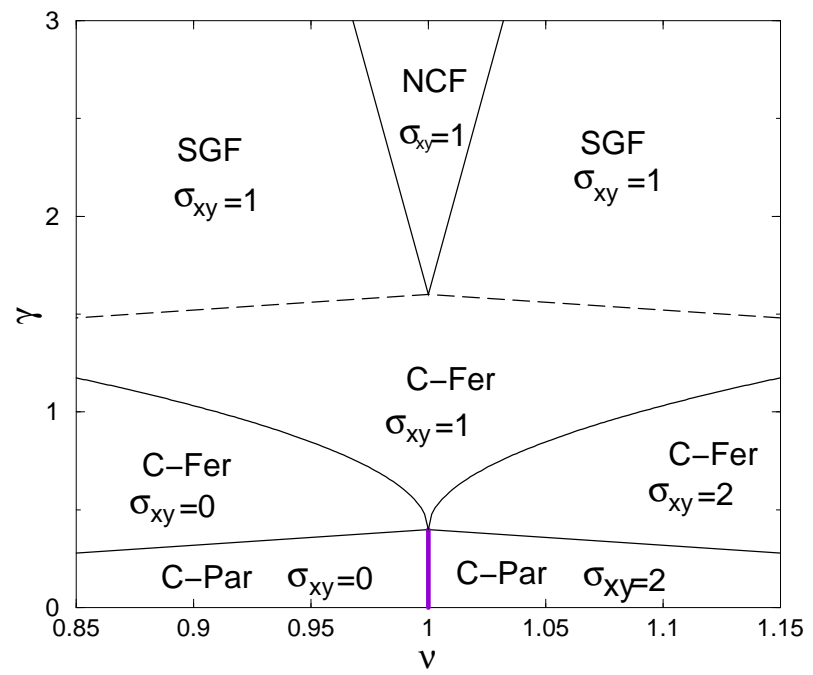

FIG. 1. Phase diagram summarizing the results. Here C-Par indicates the compressible paramagnetic ground state, C-Fer the partially polarized compressible ferromagnetic ground state, SGF the spin gapped ferromagnetic ground state, and NCF the non-collinear ferromagnetic ground state. This phase diagram is qualitative in nature and transition points vs. $\gamma$ should be taken as upper limits to any realistic phase diagram.

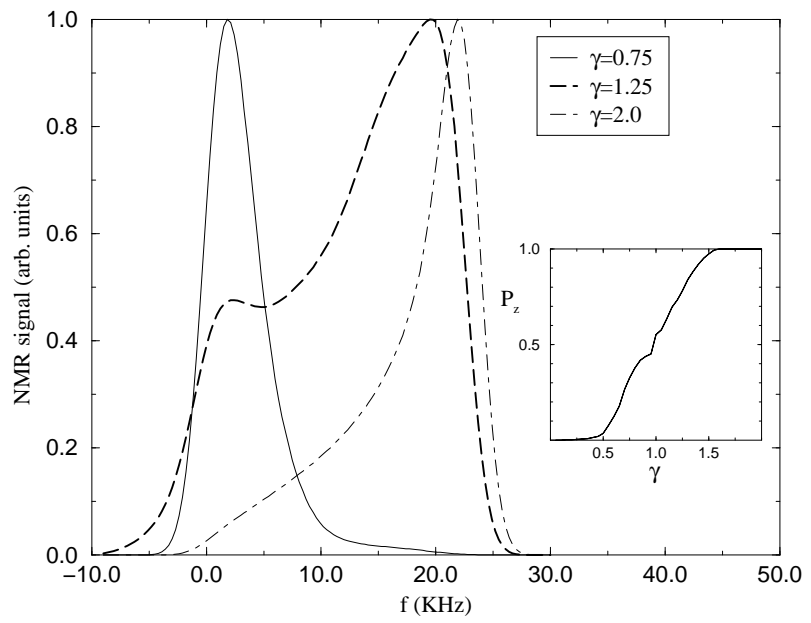

FIG. 2. NMR spectrum for $\nu=1$ and $\tilde{g}=0.015$ at different $\gamma$ 's. The insert indicates the average magnitude of the local polarization $\mathrm{P}_{\mathrm{z}}$ as a function of $\gamma$. The sample parameters correspond to ones the used in the experimental studies of Ref. [3].

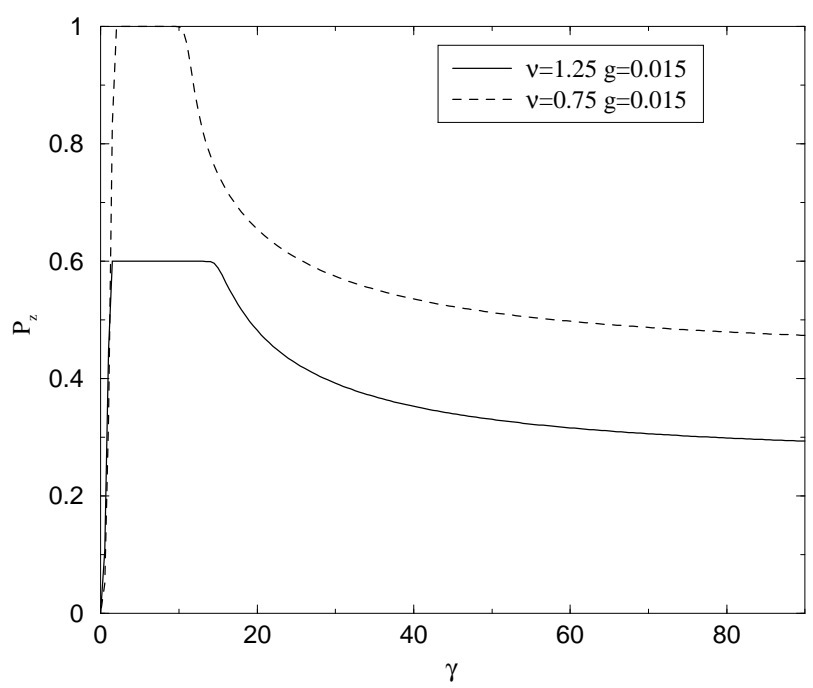

FIG. 3. Global polarization phase diagram for a fixed disorder realization. The transition to a maximally polarized state (Laughlin quasiparticle glass) occurs at $\gamma \approx 1.5-2$ for all disorder realizations obtained. The transition from a Laughlin quasiparticle glass to a Skyrmion glass occurs at $\gamma \approx 15$. Local polarization and density profiles have been given elsewhere [7]. 